Alcoholic and Diabetic Ketoacidosis: Is It Possible, with Biological Parameters, to Differentiate These Two Pathologies

F. Staïkowsky; V. Bridon Citerne; S. Carmes; A. Lepelletier, S. Huret; E. Thommeray; D. Ozouf Emergency Department, Caen, FRANCE

Introduction: Alcoholic ketoacidosis is an unrecognised syndrome in Europe. It often is unrecognised and could be very misleading. Clinicians must be aware that besides diabetic ketoacidosis, alcoholic ketoacidosis is another cause of ketoacidosis with some peculiar signs The alcoholic ketoacidosis possesses clinical and biological characteristics close to those of diabetic ketoacidosis; the processing differs and risks of an inappropriate treatment with insulin are not negligible. The purpose of this work is to extract biological serum elements on admission to an emergency room sufficiently significant to differentiate between these two pathologic states.

Methods: 26 biological blood parameters and 2 calculated values (anion gap and osmolarity) were compared in patients with alcoholic ketoacidosis and in consecutive patients with diabetic ketoacidosis admitted in an emergency room.

Results: 16 patients with alcoholic ketoacidosis (11 women and 5 men, mean age $46 \pm 12.5$ years) were compared to 29 patients with diabetic ketoacidosis ( 19 women and 10 men, mean age $44.7 \pm 22$ years). The serum rates of sodium, potassium, chloride, carbon dioxide, urea nitrogen, creatinine, total proteins, amylase, lactate dehydrogenase, creatine kinase, lactates, anion gap, and the arterial blood gases did not differ between the groups. In patients with alcoholic ketoacidosis: (1) the glucose levels and the serum osmolarity were significantly lower (glucose $8.6 \pm 5$ vs. 41 $\pm 22 \mathrm{mmol}^{-1} \mathrm{l}^{-1} p<0.001$ ); (2) the serum levels of aspartate aminotransferase, bilirubin and gamma glutamyltransferase were significant higher (aspartate aminotransferase $121.7 \pm 92.3$ vs. $30.4 \pm 22.5$ UI. $1^{-1}$; bilirubin $17.2 \pm 8.4$ vs. $7.86 \pm 4.51$ mmol..$^{-1}$; gamma glutamyltransferase 537 \pm 460.5 vs. $48 \pm 55.6$ UI. $^{-1} ; p<0.001$ ); (3) the mean counts of leukocytes, red blood cells, and platelets were significantly lower (leukocytes $11,387 \pm 4,580$ vs. $17,982 \pm 7,070$ $/ \mathrm{mm}^{3}$; red blood cells $3.97 \pm 0.45$ vs. $4.81 \pm 0.72,106 / \mathrm{mm}^{3}$; platelets $199,375 \pm 83,178$ vs. $308,607 \pm 103,507 / \mathrm{mm}^{3}$; $p$ $<0.001$ ), and the mean corpuscular volume was greater $\left(103.2 \pm 8\right.$ vs. $\left.88.3 \pm 7.7 \mu^{3} ; p<0.001\right)$. The most significant abnormal serum values in favour of alcoholic ketoacidosis were glucose $<20 \mathrm{mmol} . \mathrm{l}^{-1}$ (sensitivity and specificity $100 \%$ ), an increase of aspartate aminotransferase (sensitivity $93.7 \%$, specificity $93.1 \%$ ), and gamma glutamyltransferase (sensitivity $92.8 \%$, specificity $61 \%$ ), and a mean corpuscular volume $>98 \mu^{3}$ (sensitivity $75 \%$, specificity $96.4 \%$ ). An increase of the bilirubin and a platelets counts $<150,000$ $/ \mathrm{mm}^{3}$ were specific (respectively 100 and $92.8 \%$ ), but not sensitive (23 and 25\%).

Discussion: The diagnosis of alcoholic ketoacidosis is evoked in patients with history of alcohol abuse and without past of diabetes, with a normal, slightly elevated or sometimes decreased serum glucose. However, there are some clinical forms in which the serum glucose is increased; in these cases, the existence of others biological signs, such that an increase of aspartate aminotransferase, gamma glutamyltransferase, or mean corpuscular volume are very important in the differential diagnosis with the diabetic ketoacidosis.

Key words: alcoholic ketoacidosis; chemistries; clinical signs; diabetic ketoacidosis; differentiation

Prehosp Disast Med 2001;16(2):s68.

\section{Effect of Sodium Bicarbonate on Ventricular Conduction Velocities in Experimental Intoxication by Clomipramine in Dogs F. Stä̈kowsky; $;^{1,2}$ A. Bardou; ${ }^{1}$ D Van Euw; ${ }^{1}$ D. Ozouf; ${ }^{2}$ I. Dandine ${ }^{2}$ \\ 1.INSERM 256, Paris, FRANCE \\ 2. Emergency Department, CHU Caen, FRANCE}

Introduction: The myocardial electrical impulse does not displace to the same velocity (V) in all directions (anisotropic conduction). The purpose of this work were to study, with epicardial mapping, the influence of a tricyclic antidepressant, clomipramine, on the conduction velocity longitudinal (VL) and transverse (VT) to myocardial fiber orientation and on anisotropy $(A=V L / V T)$, and their modifications by the administration of sodium bicarbonate (B).

Methods: A plaque with 64 electrodes, positioned on the left anterior ventricular wall of nine anesthetized dogs, allowed the delivery through central electrodes, of programmed electrical stimulations that induced ventricular complexes and their collection. Each entailed unipolar electrogram was processed by a computer system that drew the isochrones and a map of activation that allowed the calculation of the V. The clomipramine was infused $(0.5$ $\mathrm{mg} / \mathrm{kg} / \mathrm{min}$. as a continuous intravenous infusion) for 75 min. At T60 (60 min.), the dogs received the sodium bicarbonate infusion until the duration of QRS complexes returned to its T0 value. A lengthening of the duration of the QRS complexes of at least $30 \%$ of their T0 value was necessary before the administration of sodium bicarbonate. Results: All values (mean arterial pressure, heart rate, QRS and QT intervals, and V) differed significantly $(p<0.05)$ compared to T0 values except QRS at T65.

$\begin{array}{lllll} & T_{0} & T_{15} & T_{30} & T_{45} \\ \text { MAP (mmHg) } & 115.8 \pm 13.6 & 99.5 \pm 19.4 & 98.8 \pm 16.4 & 92.6 \pm 7.5 \\ \text { HR (cyc/min) } & 389 \pm 59.4 & 497.5 \pm 95 & 546 \pm 80 & 575 \pm 77.6 \\ \text { QRS (ms) } & 49 \pm 6.4 & 59 \pm 9.5 & 65 \pm 10.3 & 67.5 \pm 10 \\ \text { QT (ms) } & 188 \pm 32 & 217 \pm 39 & 247 \pm 34 & 257 \pm 36 \\ \text { VL (cm/sec) } & 57.4 \pm 19 & 47.6 \pm 14 & 38.6 \pm 11 & 38.3 \pm 11 \\ \text { VT (cm/sec) } & 28.2 \pm 8 & 22.4+7 & 18.8 \pm 4 & 19.1 \pm 6 \\ \text { A } & 2.1 \pm 0.6 & 2.1 \pm 0.5 & 2.1 \pm 0.4 & 2.1 \pm 0.4 \\ & & & & \\ \text { MAP (mmHg) } & 92.5 \pm 13.3 & 82.7 \pm 18.5 & 88.2 \pm 25.6 \\ \text { HR (cyc/min) } & 611.2 \pm 80.6 & 587.5 \pm 88.7 & 595 \pm 87.4 \\ \text { QRS (ms) } & 70 \pm 10.3 & 51 \pm 6.5 & 64.4 \pm 9 \\ \text { QT (ms) } & 261 \pm 22 & 265 \pm 36 & 272 \pm 36 \\ \text { VL (cm/sec) } & 33.4 \pm 11 & 32.8 \pm 15 & 34.9 \pm 11 \\ \text { VT (cm/sec) } & 15.8 \pm 4 & 15.6 \pm 4 & 17.5 \pm 5 \\ \text { A } & 2.1 \pm 0.7 & 2.1 \pm 0.7 & 2.1 \pm 0.5\end{array}$

Conclusion: The administration of clomipramine slowed 